\title{
Neoadjuvant therapy: theoretical, biological and medical consideration
}

\author{
Evgeny N. Imyanitov ${ }^{1,2,3,4}$, Grigoriy A. Yanus ${ }^{1,2}$ \\ ${ }^{1}$ Department of Tumor Growth Biology, N.N. Petrov Institute of Oncology, St.-Petersburg 197758, Russia; ${ }^{2}$ Department of Medical Genetics, \\ St.-Petersburg Pediatric Medical University, St.-Petersburg 194100, Russia; ${ }^{3}$ Department of Oncology, I.I. Mechnikov North-Western Medical \\ University, St.-Petersburg 191015, Russia; ${ }^{4}$ Department of Oncology, St.-Petersburg State University, St.-Petersburg 199034, Russia \\ Contributions: (I) Conception and design: All authors; (II) Administrative support: None; (III) Provision of study materials or patients: None; (IV) \\ Collection and assembly of data: All authors; (V) Data analysis and interpretation: All authors; (VI) Manuscript writing: All authors; (VII) Final \\ approval of manuscript: All authors. \\ Correspondence to: Evgeny N. Imyanitov. Department of Tumor Growth Biology, N.N. Petrov Institute of Oncology, St.-Petersburg 197758, Russia. \\ Email: evgeny@imyanitov.spb.ru.
}

\begin{abstract}
Neoadjuvant therapy (NAT) is widely utilized in the routine management of cancer patients and various clinical trials for the treatment of breast, ovarian, rectal, esophageal, head and neck, lung, prostate and many other cancer types. There is a number of potential benefits of applying systemic treatment before the operation. NAT may significantly reduce the tumor burden thus allowing less traumatic surgery. NAT is often considered as personalized in vivo drug sensitivity test, as it allows rapid evaluation of tumor response to a given therapy and consequent adjustment of further treatment planning. NAT is an invaluable tool for in-human testing of novel therapeutic compounds, as it deals with yet chemonaive patients and permits pathological and molecular analysis of treatment-exposed tumor tissues. There are also some arguments against the use of NAT. The delay of surgery may increase the chances for metastatic tumor spread. NAT often results in the selection of treatment-resistant tumor clones, which cannot be eliminated by subsequent adjuvant therapy. NAT is likely to compromise visual inspection of surgical wound, as it reduces the size of tumor lumps and makes them invisible for the operating physician. Animal models cannot fully recapitulate the complexity of human cancer biology, the sophistication of modern surgical interventions, and the spectrum of tumor drug sensitivity in neoadjuvant and adjuvant settings. Therefore, studies on the rationale for NAT are largely limited to human studies.
\end{abstract}

Keywords: Neoadjuvant therapy (NAT); pathologic complete response (pCR); treatment resistance; review

Submitted Aug 10, 2018. Accepted for publication Sep 26, 2018.

doi: $10.21037 /$ cco.2018.09.05

View this article at: http://dx.doi.org/10.21037/cco.2018.09.05

The term "neoadjuvant therapy" (NAT) is usually applied to a number of therapeutic modalities, which are administered before the surgical intervention in order to reduce tumor volume. Historically, preoperative therapy was used to convert inoperable tumor into surgically curable condition. In these situations, the physician actually does not have a choice between upfront surgery or therapy, therefore the use of non-surgical methods is the only possible option. If the therapy works well, the feasibility of consequent tumor removal becomes an issue for consideration: in some oncological disciplines surgery is regarded is an integral part of the treatment plan (1), while for other varieties of cancer the resection of residual tumor lumps is uncommon or perceived as a part of experimental medical science (2).

It is often suggested to use more restrictive definition of NAT, i.e., to consider only those clinical situations, where the gross tumor mass can technically be excised, even for the price of organ loss or excessive perioperative risks, but the use of NAT remains just a clinical option. For a few decades, radiotherapy was the only therapeutic modality, 
Table 1 Pros and cons for neoadjuvant therapy and primary surgery

\begin{tabular}{|c|c|c|}
\hline Pros and cons & Neoadjuvant therapy & Primary surgery followed by adjuvant therapy \\
\hline \multirow{3}{*}{ Advantages } & $\begin{array}{l}\text { May reduce the extent of surgery (preservation of } \\
\text { the affected organ; lowering the risk of perioperative } \\
\text { morbidity) }\end{array}$ & $\begin{array}{l}\text { Reliable visual inspection of the tumor spread during } \\
\text { surgery }\end{array}$ \\
\hline & $\begin{array}{l}\text { NAT allows to evaluate short-term response to a given } \\
\text { therapy and change the treatment scheme if alternative } \\
\text { options are available }\end{array}$ & $\begin{array}{l}\text { Strong experimental evidences for potential efficacy of } \\
\text { adjuvant therapy }\end{array}$ \\
\hline & Early control of systemic cancer disease & \\
\hline \multirow{3}{*}{ Disadvantages } & $\begin{array}{l}\text { NAT is usually mutagenic, therefore it may facilitate } \\
\text { tumor evolution }\end{array}$ & $\begin{array}{l}\text { Adjuvant therapy is often delayed due to perioperative } \\
\text { complications }\end{array}$ \\
\hline & $\begin{array}{l}\text { May result in selection and expansion of resistant tumor } \\
\text { clones }\end{array}$ & Adjuvant therapy is given without prior in vivo test \\
\hline & $\begin{array}{l}\text { Small tumor foci may be missed during the surgery and } \\
\text { therefore remain in the body }\end{array}$ & \\
\hline
\end{tabular}

which could be applied before the surgical intervention, so the earlier NAT studies were limited to the evaluation of preoperative tumor irradiation (3). The invention of drugbased therapy resulted in spectacular diversification of NAT options, bringing NAT virtually to all areas of oncology. Nowadays, NAT is utilized in clinical or investigational setting for the treatment of breast, ovarian, rectal, esophageal, head and neck, lung, prostate and many other cancer types (4-10). No animal model can fully recapitulate the complexity of human tumor biology, real-life cancer therapy and tumor surgery, therefore most of knowledge related to the clinical use of NAT arrives from the common sense and various clinical experiences.

Several assumptions underlie the utilization of NAT (Table 1). First, NAT provides an opportunity for an organsparing surgery. Most of systematic NAT trials were performed on breast cancer patients, where the purpose of organ preservation was served mainly for cosmetic reasons (4). For many other cancer types the prevention of the loss of the affected organ is absolutely vital for the quality of life or for the long-term life expectancy of the patients: the examples include cancers of larynx, sarcomas of the limb, lung cancers etc. (7-9). Secondly, NAT may allow more safe surgical intervention. For example, many patients with advanced ovarian cancer may theoretically undergo complete tumor resection. However, if the tumor spread is extensive and/or the general condition of the patient is poor, the risk of perioperative mortality may outweigh all potential benefits. Furthermore, postsurgical complications usually result in the delay of the adjuvant therapy, which negatively impacts treatment outcomes (5). Thirdly, NAT is often advertised as in vivo test for drug sensitivity: the proponents of this viewpoint suggest, that if the tumor does not respond to the therapy in the very beginning of the treatment, there is still an option to change the therapeutic scheme (11). Instead, when surgery is applied upfront, many patients are likely to receive de facto inefficient adjuvant therapy, given that the administration of the latter is based not on the individual basis but on statistical considerations of the probability of the effect. Fourthly, there is a belief, 
that early therapeutic intervention may decrease the risk of distant metastases, by eliminating circulating tumor cells and interfering with the viability of the cells within primary tumor (12).

There are also sound arguments supporting the choice in favor of the upfront surgery in potentially operable tumors (Table 1). The removal of the gross tumor bulk dramatically decreases the number of tumor cells persisting in the body. Consequently, the probability of the emergence of drugresistant clones is believed to be decreased in the same proportion. As mentioned above, the visual inspection of the tumor bed is likely to be more reliable, as preceding treatment does convert tumor lumps into invisible foci. The application of full-dose therapy after the surgery may be particularly efficient if only residual amounts of tumor cells are left in the body $(5,13)$.

In addition to purely clinical considerations, the use of NAT is influenced by the demands of medical research. Patients undergoing NAT are by definition treatment-naïve, therefore the evaluation of novel drugs in these subjects can be particularly informative. In case of drug-sensitive cancer diseases, short-term NAT is a preferred approach for the human trials even from the ethical perspective. Indeed, tumors undergo profound alterations in their biology during conventional treatment, so their profile of drug sensitivity may change to the opposite upon drug-induced selection of resistant clones. For example, tumor arising in BRCA1 germ-line mutation carriers are platinum-sensitive in the chemonaive state, but even a short-term therapy results in the selection of clones with restored BRCA1 function (14). Accordingly, there is no biological rationale to evaluate new platinum compounds or PARP inhibitors in the second or third treatment lines, given that cisplatin or carboplatin are the gold standard for the first-line treatment. If one limits clinical trials of novel agents by heavily pretreated patients, there is a high probability that potentially efficient drugs will be missed by this approach. Evaluation of new compounds in the first-line therapy for metastatic cancer is complicated when some reasonably efficient standard options are available. NAT provides a good balance, where the experimental therapy can be subjected to a meaningful and rapid clinical assessment without significant risk of compromising the overall treatment outcomes.

In addition to straightforward evaluation of tumor responsiveness to a given therapy, there is an opportunity to analyze treatment-exposed tumor and normal tissues by pathological and molecular tools (15). These considerations led to the inclusion of NAT trials in the FDA approval guidelines (16). It is also essential to consider, that NAT is likely to set somewhat higher standards for the acceptance of novel drugs. While clinical studies involving heavily pretreated patients are considered successful even if the survival in the experimental arm statistically exceeds the one in control arm just for a few days (17), many NAT trials define the pathologic complete response (pCR) as the only meaningful end-point. Although pCR is very difficult to achieve in some tumor types, for example in estrogen receptor positive breast cancer or in ovarian cancer, it is considered as a reliable indicator of complete elimination of tumor cells from the body and is strongly associated with the cure from cancer $(5,18)$. Therefore, treatments resulting in high rates of $\mathrm{pCR}$ deserve the utmost attention from research community and clinical specialists.

There are some general considerations regarding the role of therapeutic and surgical interventions, which are frequently discussed in the context of NAT, but could be interpreted both in favor of NAT and in favor of upfront surgery. Earlier oncological concepts relied on the linear model of cancer progression, where localized tumor is formed first, then metastasizes into regional lymph nodes and only then spreads to distant organs. Furthermore, canonical views on cancer pathogenesis considered tumor progression being almost entirely independent from the host factors. These assumptions have been subjected to significant revision during recent decades (19).

For example, it is beyond any reasonable doubt that micrometastases are often already present in the body even if the tumor is diagnosed on the early stage (19). Furthermore, metastatic cells may significantly differ in their properties from the cells residing in the primary tumor (20). It is unclear how these evidences may affect the choice between NAT and surgery. One could expect that even if the primary tumor undergoes shrinkage upon NAT, it does not directly imply that the same treatment will be equally effective in controlling distant disease. Furthermore, given that metastatic seeds in distant organs are present in a subset of patients at the time of surgery, it is unclear whether these patients could be reliably identified by available diagnostic tools, and how the mere presence of detectable micrometastatic disease would affect the sequence between surgery and therapy.

There are multiple evidences indicating that cancer cells dying from effective treatments, e.g., from irradiation or anticancer drugs, may somehow stimulate the growth of the remaining malignant clones, for example by secreting some tumor promoting factors $(21,22)$. On a similar note, 
multiple studies suggest that tumor microenvironment can be significantly altered by cancer therapy; these modifications are not neutral for the cancer growth, but can either speed up or slow down dissemination and proliferation of transformed cells (23-25). It is unclear how consideration of the status of tumor microenvironment may influence the choice between the NAT and the upfront surgery.

Surgery is often accompanied by massive dislodgement of cancer cells from primary tumor lump. Furthermore, surgical intervention was long suspected to stimulate the growth of otherwise dormant cancer cells (24-28). Recent research demonstrates, that the trauma-induced inflammation plays a primary role in this phenomenon (28). It is intuitively appealing to expect, that the tumor promoting effect is more likely in case of extensive surgery, that supporting the role of NAT which could minimize surgical trauma. However, there is a scarcity of experimental or clinical evidence supporting this oversimplified assumption.

Recent developments in cancer medicine may lead to revision of the concept of neoadjuvant and adjuvant treatment. All treatments applied before mid 2000s were largely non-specific, thus the inability of radiotherapy or cytotoxic drugs to completely eradicate all tumor clones seemed to be an intrinsic limitation of these modalities. Recent decade provided a number of highly efficient drugs targeted against mutated kinases (EGFR, ALK, BRAF etc.). The inability of these drugs to cure advanced cancer can be easily explained by the emergence of drug-resistant clones within the gross tumor bulk. However, limited long-term clinical efficacy of these drugs in adjuvant or neoadjuvant setting is puzzling, as small amounts of disseminated mutation-bearing cells should, in theory, die within the very first days of targeted therapy (29). The role and the optimal timing of surgery in the treatment of localized and advanced mutation-positive cancers remains to be investigated.

Novel genetic technologies provide tools for the efficient detection of single transformed cells. There is an ongoing debate whether the surgery has to be performed in patients demonstrating pCR after NAT (30). The ironic reality is that the mere fact of pCR can be established only on the basis of thorough surgery, followed by comprehensive morphological inspection of the excised tissues. Nextgeneration sequencing (NGS), polymerase chain reaction and some other methods can identify single mutated cells in the presence of excess of normal tissues (31). It is very attractive to develop diagnostic procedures, which would allow to identify patients with pCR before the surgery, and perhaps avoid unnecessary operation in a subset of cases.

\section{Acknowledgements}

Funding: This work has been supported by Russian Science Foundation (grant 16-45-02011).

\section{Footnote}

Conflicts of Interest: The authors have no conflicts of interest to declare.

\section{References}

1 Aigner F, Pratschke J, Schmelzle M. Oligometastatic Disease in Colorectal Cancer - How to Proceed? Visc Med 2017;33:23-8.

2. Salama JK, Chmura SJ. Surgery or ablative radiotherapy for breast cancer oligometastases. Am Soc Clin Oncol Educ Book 2015:e8-15.

3. O'Brien PH. Preoperative irradiation in cancer therapy. Surg Clin North Am 1971;51:61-8.

4. Untch M, Konecny GE, Paepke S, et al. Current and future role of neoadjuvant therapy for breast cancer. Breast 2014;23:526-37.

5. Leary A, Cowan R, Chi D, et al. Primary Surgery or Neoadjuvant Chemotherapy in Advanced Ovarian Cancer: The Debate Continues.... Am Soc Clin Oncol Educ Book 2016;35:153-62.

6. Bringeland EA, Wasmuth HH, Grønbech JE. Perioperative chemotherapy for resectable gastric cancer - what is the evidence? Scand J Gastroenterol 2017;52:647-53.

7. Harrison DJ, Schwartz CL. Osteogenic Sarcoma: Systemic Chemotherapy Options for Localized Disease. Curr Treat Options Oncol 2017;18:24.

8. Bhattacharyya T, Kainickal CT. Current Status of Organ Preservation in Carcinoma Larynx. World J Oncol 2018;9:39-45.

9. Lewis J, Gillaspie EA, Osmundson EC, et al. Before or After: Evolving Neoadjuvant Approaches to Locally Advanced Non-Small Cell Lung Cancer. Front Oncol 2018;8:5.

10. Pignot G, Maillet D, Gross E, et al. Systemic treatments for high-risk localized prostate cancer. Nat Rev Urol 2018;15:498-510.

11. Kim R, Osaki A, Toge T. Current and future roles of neoadjuvant chemotherapy in operable breast cancer. Clin 
Breast Cancer 2005;6:223-32.

12. Goodman KA. Total neoadjuvant therapy for rectal cancer. Cancer Radiother 2018;22:459-65.

13. Martin DS. The scientific basis for adjuvant chemotherapy. Cancer Treat Rev 1981;8:169-89.

14. Sokolenko AP, Savonevich EL, Ivantsov AO, et al. Rapid selection of BRCA1-proficient tumor cells during neoadjuvant therapy for ovarian cancer in BRCA1 mutation carriers. Cancer Lett 2017;397:127-32.

15. Bardia A, Baselga J. Neoadjuvant therapy as a platform for drug development and approval in breast cancer. Clin Cancer Res 2013;19:6360-70.

16. Network FDA. U.S. Food and Drug Administration. Available online: https://www.fda.gov/downloads/Drugs/ GuidanceComplianceRegulatoryInformation/Guidances/ UCM305501.pdf

17. Stewart DJ, Kurzrock R. Cancer: the road to Amiens. J Clin Oncol. 2009;27:328-33.

18. Cortazar P, Zhang L, Untch M, et al. Pathological complete response and long-term clinical benefit in breast cancer: the CTNeoBC pooled analysis. Lancet 2014;384:164-72.

19. Fisher B. Biological and clinical considerations regarding the use of surgery and chemotherapy in the treatment of primary breast cancer. Cancer 1977;40:574-87.

20. Hosseini H, Obradović MM, Hoffmann M, et al. Early dissemination seeds metastasis in breast cancer. Nature 2016;540:552-8.

21. Obenauf AC, Zou Y, Ji AL, et al. Therapy-induced tumour secretomes promote resistance and tumour progression. Nature 2015;520:368-72.

22. Gerweck LE, Wakimoto H. At the Crossroads of Cancer

Cite this article as: Imyanitov EN, Yanus GA. Neoadjuvant therapy: theoretical, biological and medical consideration. Chin Clin Oncol 2018;7(6):55. doi: 10.21037/cco.2018.09.05
Stem Cells, Radiation Biology, and Radiation Oncology. Cancer Res 2016;76:994-8.

23. Barker HE, Paget JT, Khan AA, et al. The tumour microenvironment after radiotherapy: mechanisms of resistance and recurrence. Nat Rev Cancer 2015;15:409-25.

24. Ebos JM. Prodding the Beast: Assessing the Impact of Treatment-Induced Metastasis. Cancer Res 2015;75:3427-35.

25. Martin OA, Anderson RL, Narayan K, et al. Does the mobilization of circulating tumour cells during cancer therapy cause metastasis? Nat Rev Clin Oncol 2017;14:32-44.

26. Horowitz M, Neeman E, Sharon E, et al. Exploiting the critical perioperative period to improve long-term cancer outcomes. Nat Rev Clin Oncol 2015;12:213-26.

27. Tohme S, Simmons RL, Tsung A. Surgery for Cancer: A Trigger for Metastases. Cancer Res. 2017;77:1548-52.

28. Krall JA, Reinhardt F, Mercury OA, et al. The systemic response to surgery triggers the outgrowth of distant immune-controlled tumors in mouse models of dormancy. Sci Transl Med 2018;10.

29. Zhai H, Zhong $W$, Yang $X$, et al. Neoadjuvant and adjuvant epidermal growth factor receptor tyrosine kinase inhibitor (EGFR-TKI) therapy for lung cancer. Transl Lung Cancer Res 2015;4:82-93.

30. van la Parra RF, Kuerer HM. Selective elimination of breast cancer surgery in exceptional responders: historical perspective and current trials. Breast Cancer Res 2016;18:28.

31. Sokolenko AP, Imyanitov EN. Molecular diagnostics in clinical oncology. Front Mol Biosci 2018;5:76. 